ISSN: 0123-50-95

E-ISSN: $2389-9441$

Vol. 5 - $\mathrm{N}^{\circ} 25$

Julio- Diciembre, año 2019

pp. $37-61$

\title{
La pregunta por los fundamentos epistemológicos de la investigación en Educación -aportes para una discusión epistemológica-
}

The question about epistemological foundations of the research in education -contributions for an epistemological discussion-

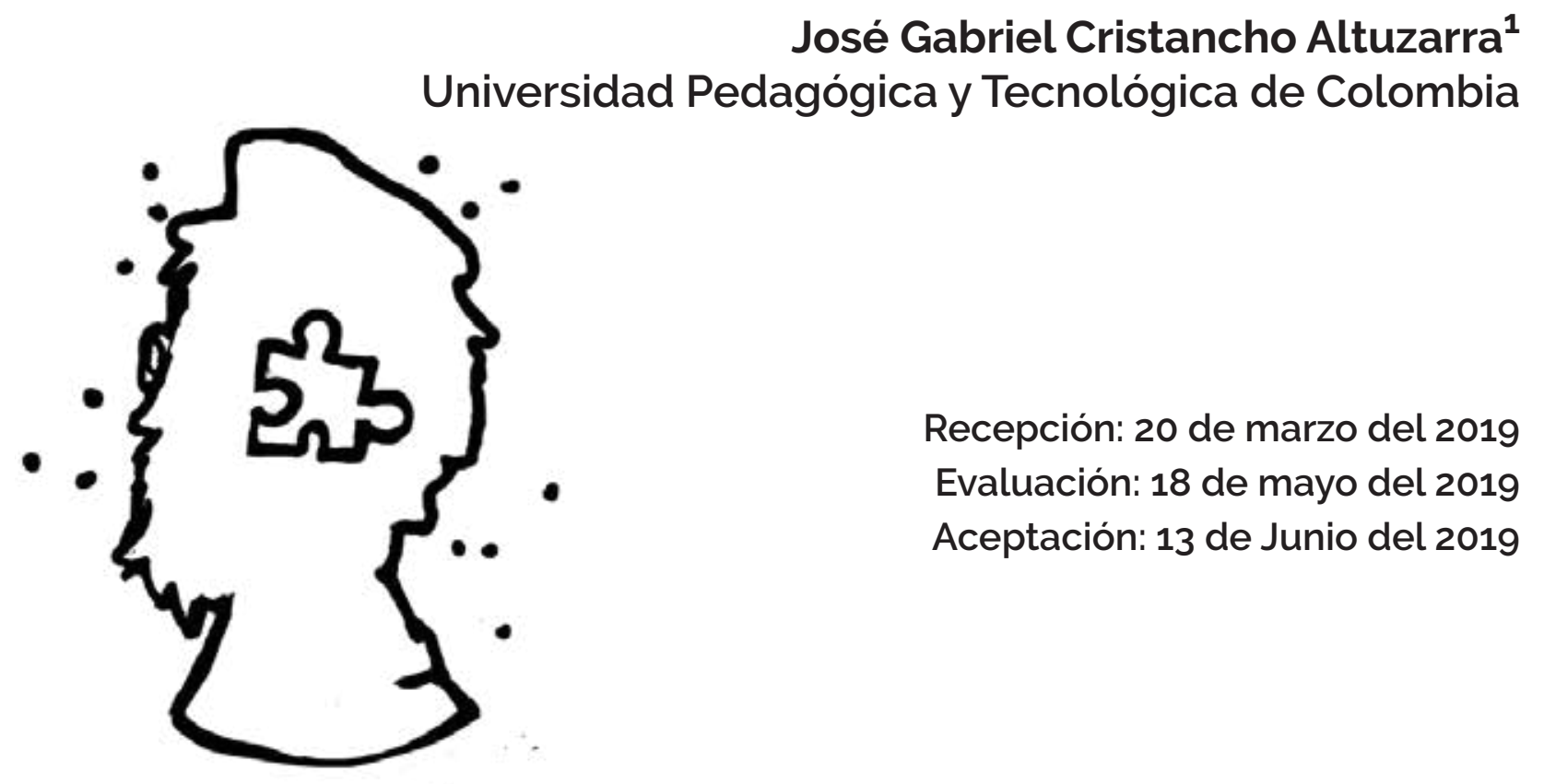

1 Doctor en Educación. Docente Investigador de la Universidad Pedagógica y Tecnológica de Colombia y de la Universidad Pedagógica Nacional. Correo electrónico: jose.cristanchoo1@uptc.edu.co 


\section{Resumen:}

La epistemología es un campo de reflexión fundamental para la construcción del conocimiento. Por esta razón, en este artículo se explicitan las preguntas fundamentales que subyacen a la investigación en educación y sus pretensiones de cientificidad, tomando como base que se propone comprender las cuestiones epistemológicas implícitas en la construcción de conocimiento en el campo de la educación y la importancia de su determinación para su desarrollo. Luego de contextualizary justificar la cuestión, el trabajo se divide en tres partes con un método deductivo; en la primera se ubica la cuestión de la epistemología mostrando los conceptos de ciencia más importantes que ha habido a lo largo del tiempo; luego se ponen en evidencia las constantes o mínimos que todo conocimiento riguroso parece requerir; en la tercera parte se plantea la pregunta por la investigación en educación como campo específico.

Palabras clave: Educación, investigación, epistemología de las ciencias sociales, epistemología de la investigación educativa

\section{Abstract:}

Epistemology is a field of fundamental reflection for the construction of knowledge. For this reason, in this article, we make explicit the fundamental questions that underlie the research in education and its claims of being scientific, based on the proposal for understanding the epistemological questions implicit in the construction of knowledge in the field of education and the importance of determining its development. After a contextualization and justification of the question, the work divides itself into three pieces with a deductive method. The first piece contains the question of epistemology showing the most important concepts of science that have appeared over time. Afterward, constant or minimum aspects that every rigorous knowledge appears to demand. In the third piece, we state the question about research in education as a specific field.

Keywords: education, research, epistemology of social sciences, epistemology of educative research. 


\section{La question par les fondements épistémologiques de la recherche en Éducation : contributions pour une discussion épistémologique.}

\section{Résumé:}

L'épistémologie est un domain de réflexion fondamentale pour la construction des connaissances. Par cette raison, dans cet article seront expliquées les questions fondamentales qui sont sous-jacentes dans la recherche éducative et leurs ambitions de scientificité ; en s'adressant vers la compréhension des questions épistémologiques implicites dans la construction de connaissances dans le domain de l'éducation et l'importance de leur détermination pour mener à bien leur développement. Après avoir contextualisé et justifie le point central, le travail se divise en trois partes avec une méthode déductive ; dans la première parte s'ubique la question épistémologique, montrant les concepts de science les plus importants qui ont existé au fils du temps ; ensuite, les constants détails existants ou non dans toute rigoureuse connaissance seront mis en évidence ; en guise de conclusion, on abordera la question par la recherche en éducation comme domain spécifique.

Mots-clés : Éducation, recherche - investigation, épistémologie des sciences sociales, épistémologie de l'investigation - recherche éducative. 


\section{A pergunta pelos fundamentos epistemológicos da pesquisa em Educação: aportes para uma discussão epistemológica}

\section{Resumo:}

A epistemologia é um campo de reflexão fundamental para a construção do conhecimento. Assim, neste artigo explicitam-se as perguntas fundamentais que subjazem na pesquisa em educação e suas pretensões de cientificidade, tomando como base propor e compreender as questões epistemológicas implícitas na construção do conhecimento no campo da educação e a importância de sua determinação para seu desenvolvimento. Após de contextualizar e justificar a questão, o trabalho se divide em três partes com um método dedutivo; na primeira localiza-se a questão da epistemologia mostrando os conceitos de ciência mais importantes que houve no percorrer do tempo; logo se colocam em evidência as constantes ou mínimos que todo conhecimento rigoroso parece requerer; na terceira parte se propor a pergunta pela pesquisa em educação como campo específico.

Palavras-chave: educação, pesquisa, epistemologia das ciências sociais, epistemologia da pesquisa educativa. 


\section{Introducción}

Existen dos conceptos y campos de acción que están necesariamente relacionados entre sí: la educación y la investigación. En efecto, lo que los une y los interseca es que en ambos se busca la construcción de conocimiento.

La investigación, que surge, entre otras cosas, del deseo de saber, de construir conocimiento, implica problematizar, cuestionar, formular preguntas, descubrir problemas, esclarecerlos y al mismo tiempo profundizar en un campo de conocimiento ${ }^{2}$.

Entre tanto, en la educación la construcción de conocimiento se da en virtud de la interacción que implica la enseñanza y el aprendizaje. Esta interacción puede ser deliberada o no. Y también puede ser una educación doctrinaria, alienante, acrítica, en la que el profesor o docente es sólo un transmisor y sus estudiantes son pasivos, con lo cual la construcción de conocimiento puede quedar en peligro.

Por eso la investigación es quizás, la manera más eficaz de propiciar que los sujetos se empoderen gracias al desarrollo de habilidades analíticas e interpretativas que implican la reflexión activa y la acción reflexiva. En suma, si la investigación es el núcleo, la materia y la forma de la educación, ésta sería condición de posibilidad de cuestionamiento, comprensión, crítica y creación de conocimiento. $Y$ en ese marco, el docente seria un educador en un sentido amplio de la palabra, es decir, un trabajador de la cultura, un intelectual que piensa y reflexiona su contexto y el de su comunidad, es decir, un investigador.

El conocimiento y su construcción es uno de los objetos de análisis de la epistemología. Y esta es la razón fundamental por la cual es necesario volver la mirada sobre esta cuestión. En el trasfondo de esas cuestiones hay una pregunta más amplia y compleja: ¿Qué es el conocimiento, en el sentido más estricto y riguroso del término? ¿Cuáles son las condiciones de posibilidad de esa forma de conocimiento? En sentido general, ciencia ha sido el concepto que se ha usado para designar

2 En el mundo contemporáneo, investigación se dice al menos de tres maneras: la investigación periodistica, la investigación criminalista y la investigación académica o científica; cada una de ellas tiene unos propósitos y modos de proceder especificos. Aqui nos referimos a la investigación académica o científica que, en cierto modo, es la matriz de las otras. 
el conocimiento más riguroso y estricto. Por eso, la pregunta por la ciencia, las preguntas anteriormente subrayadas, son, en términos generales, las preguntas fundamentales de la epistemología y se extienden a cada una de las disciplinas o campos de estudio.

Ahora bien, ¿qué relación hay entre la epistemología y la educación? Hay tres relaciones fundamentales:

a. en la educación básica y media hay una formación inicial en cada ciencia (matemáticas, ciencias naturales, ciencias sociales, etc.); como la epistemología fundamenta las ciencias - ¿qué hace que la matemática sea una ciencia? ¿Por qué las ciencias naturales son ciencias?, etc. -, tener claridad de los principios epistemológicos fundamentales de toda disciplina ayudaría a enseñar y aprender mejor cada una de ellas;

b. la pedagogía y la didáctica son campos de estudio que se preguntan por las condiciones de posibilidad de la enseñanza y el aprendizaje; por tanto, la epistemología ayuda a la pedagogía y a la didáctica a fundamentarse como campos de conocimiento;

c. la investigación educativa es un campo de estudio especifico y como tal también requiere fundamentarse epistemológicamente; por tanto, la epistemología se requiere para ejercerla, vale decir, para que el trabajo de grado en una maestría en educación merezca el nombre de conocimiento, en el sentido más estricto y riguroso del término.

En este marco, este escrito se propone hacer explícitas las preguntas fundamentales que subyacen a la investigación en educación y sus pretensiones de cientificidad. Para tal fin el texto se divide en tres partes con un método deductivo, yendo de lo general a lo particular; en la primera se ubica la cuestión de la epistemología mostrando los conceptos de ciencia más importantes que ha habido a lo largo del tiempo; en el segundo se ponen en evidencia las constantes o mínimos que todo conocimiento riguroso parece requerir; en la tercera parte se plantea la pregunta por la investigación en educación como campo específico atinente a lo que nos interesa. 


\section{La pregunta por la ciencia y el conocimiento científico}

La epistemología ha sido definida usualmente como el campo de reflexión que se pregunta qué es ciencia, qué conocimiento lo es y cuáles son las condiciones de posibilidad del conocimiento científico. En efecto, episteme es un término griego que se ha traducido como ciencia. En la actualidad, se ha hecho recurrente creer que la ciencia es sinónimo de verdad, de veracidad, de exactitud. En la vida cotidiana, decir que algo está "científicamente comprobado" es sinónimo de que es un conocimiento indudable, válido y universal.

Pero el asunto no es así de simple: primero que todo, el concepto de ciencia ha cambiado a lo largo del tiempo; y, en segundo lugar, toda teoría científica está sometida a procesos de discusión y validación; así como la teoría de la relatividad de Einstein replanteó algunos principios de la física de Newton, una teoría que hoy es considerada válida, puede no serlo dentro de 20 ó 30 años.

Por eso, para ver con más detenimiento esta situación, veamos algunas de las modificaciones más profundas que ha tenido la categoría ciencia a lo largo del tiempo; me referiré sólo a cuatro circunstancias, de una forma esquemática: en primer lugar podemos identificar un concepto de ciencia desde el s. IV a.c. En pensadores griegos como Sócrates, Platón, Aristóteles, y en pensadores como Cicerón y San Agustín, Santo Tomás, entre otros, el vocablo griego episteme y el latino scientia, designaban la ciencia.

Para los griegos, la episteme se oponía a la doxa, es decir a la opinión; una opinión es un punto de vista sobre algo, lo que se nos ocurre, lo que creemos, lo que suponemos, pero que no está respaldado por nada; ni por argumentos sólidos, es decir, algo de lo cual no podemos dar razones lógicas; la episteme sería todo lo contrario: un conocimiento del cual se pueden dar las razones, las explicaciones y sus fundamentos.

De algún modo, el aporte más importante que hicieron los griegos fue el desarrollo de la lógica, como parte de una formación cultural y política basada en el análisis, el discernimiento y la producción de argumentos: gracias a ello, cualquier punto de vista se sometía a crítica y no cualquier cosa podía admitirse. 
Más tarde, para el cristianismo, la scientia fue entendida a la vez como una cualidad adquirida o concedida al ser humano, un don de Dios, concedido por la revelación. En este caso, a diferencia de los griegos, la fe, la autoridad eclesiástica, ayudados con las reglas de la lógica ${ }^{3}$ se volvieron los criterios más importantes para validar el conocimiento.

En todo caso, en estos primeros conceptos de ciencia, hay dos puntos en común: era un concepto singular, es decir, se hablaba de La Ciencia, no de ciencias, en plural; y en segundo lugar, se entendía como la totalidad del conocimiento de las causas y los principios, bien fuera fundamentados en la lógica y la razón (el logos griego) o en la verdad revelada por Dios (la fe cristiana).

Este concepto de ciencia predominó hasta el s. XV; poco a poco apareció un segundo momento: la Iglesia fue perdiendo paulatinamente su hegemonia y se cuestionó su autoridad cultural y política; se empezó a usar más la observación para comprender los fenómenos y aparecieron nuevas teorias astronómicas y sociales; se generó la discusión sobre las condiciones de posibilidad del conocimiento.

En este segundo momento, la modernidad, las autoridades y tradiciones fueron vistas con sospecha, la fe fue considerada superstición y asi fueron excluidas de la ciencia; se desconfió de los sentidos y la experiencia de la vida cotidiana; y la lógica y el razonamiento por sí solos, fueron pensados como mera especulación vacía de sentido.

No obstante, Newton marcó la diferencia: aplicó las reglas matemáticas a la observación de los fenómenos y logró calcular con exactitud la distancia entre el sol y la tierra, entre otros fenómenos que para el momento parecían imposibles; con este método corroboró la teoría heliocéntrica de Copérnico, Képler y Galileo y pudo extender los conocimientos sobre la mecánica, el movimiento y otras propiedades de los cuerpos.

Para ese entonces, muchos creyeron que Newton habia logrado hacer de la física una ciencia "perfecta": era exacta, y se corroboraba en la experiencia. Por eso, Kant,

3 De hecho, uno de los aportes de la Edad Media a la historia de la ciencia fue el desarrollo de la lógica; gracias a estos desarrollos los modernos y luego los contemporáneos tuvieron las bases para desarrollar la lógica matemática, la cual fundamentaría los sistemas informáticos. 
viendo en este ideal postuló una de las teorias epistemológicas más importantes que hasta cierto punto sigue marcando a la ciencia contemporánea: el conocimiento es ciencia cuando se ciñe a las reglas de la razón, tiene referentes en la experiencia y es condición de posibilidad de que ese conocimiento siga aumentando.

Esto tendría efectos paulatinos: la religión sería declarada superstición; la metafísica, mera especulación; y la ciencia ya no sería tanto una cualidad, sino un conjunto sistemático de conocimientos en continua expansión gracias a unos procedimientos metódicos.

Además la ciencia dejó de ser una cosa unitaria y total; apareció el concepto de ciencias, en plural, para designar disciplinas acotadas con objetos de estudio específicos o delimitados. Desde entonces, el paradigma experimentaly matemático se hizo prevaleciente y colocó en punta las ciencias exactas, naturales y aplicadas; y aparecería la estadística, la "ciencia del Estado", aquella dedicada a aplicar la matemática para hacer seguimiento de los fenómenos sociales demográficos, económicos, sociales, etc.

Siuna disciplina queria serciencia debia usarmétodos experimentalesymatemáticos; de lo contrario no lo era; esto afectó mucho a la pedagogía, por ejemplo, porque siempre se le criticó que no era ciencia; para reivindicar su estatus epistemológico, tuvo que hacerse siempre al lado de otras disciplinas, como la psicología; esta es la matriz epistemológica de la psicopedagogía y de todas las teorias pedagógicas que tienen como autores de cabecera a psicólogos.

Esta concepción de ciencia aportó que el conocimiento fuese además de lógico, exacto y con incidencias en la práctica; ya no bastaba con argumentar, como para los griegos; se requeria el método experimental; no obstante, implicó negar los saberes de las personas del común, de las personas analfabetas o de las personas de otras culturas y sociedades marginadas, como los indígenas y las mujeres o a todos aquellos excluidos de la educación; la ciencia se volvió un asunto exclusivo de una élite, a saber, los "expertos"; se consideró que sólo ellos investigan y pueden hacerlo. 
El tercer momento se da desde el s. XIX y en el s. XX; el concepto de ciencia de la modernidad sufrió serios cuestionamientos: destacamos tres movimientos: el primero, el más radical, fue planteado por los pensadores alemanes Nietzsche y Freud: para ellos la razón humana no era el fundamento del ser humano; para ellos la razón es una tela que recubre una maraña de instintos y pulsiones irracionales del ser humano; desde este punto de vista, para Nietzsche la ciencia no sería la máxima perfección humana sino una herramienta más para sobrevivir y ejercer el poder sobre otros.

El segundo movimiento no fue tan radical; pensadores que querian investigar desde otros métodos cuestionaban que el ser humano y lo social fueran medibles y exactos como los fenómenos naturales, porque implicaba pensar al ser humano como predecible, manipulable; así, serian criticables las teorias conductistas y la experimentación con humanos, cuya base epistemológica es la ciencia experimental como modelo.

Los alemanes Heidegger y Gadamer, tomaron como ejemplo el arte; para ellos no es que el arte sea simplemente otra forma de acceder al conocimiento; el arte y la ciencia son formas de dar sentido: el ser humano no conoce; el ser humano da sentido. Por eso las cuestiones de la epistemología dominante (cuáles son las condiciones de posibilidad de la ciencia como conocimiento verificable y exacto) querian ser desplazadas por la de la ciencia de la interpretación, es decir, la hermenéutica (cuya pregunta es cómo es posible la comprensión).

A esta crítica se le denominó giro hermenéutico e hizo énfasis en la posibilidad de métodos interpretativos, que se complementaron luego con la etnografía y la antropología y la historia cultural. Este aporte hizo posible que la pedagogía y la didáctica tuvieran un espacio alternativo para ser consideradas como disciplinas y conocimientos legítimos. Además, por este aporte se reconoció y reivindicó el conocimiento de personas tradicionalmente excluidas o personas analfabetas, no letradas o que han construido su conocimiento sin la mediación de la escritura.

Cabe aclarar que este segundo movimiento tiene dos tendencias: los que reconocen que las comunidades analfabetas o no letradas tienen conocimientos valiosos pero 
piensan que ellas son inexpertas; así, sigue habiendo una concepción implícita de que los investigadores son expertos y las comunidades son objeto de investigación, meros informantes; este criterio es cercano al de la ciencia experimental, solo que con métodos interpretativos. Esta tendencia ha sido usada también en la investigación pedagógica y didáctica.

La segunda tendencia fue propuesta por el colombiano Orlando Fals Borda: la investigación acción participante: no sólo se reconoce que las comunidades analfabetas o no letradas tienen conocimiento valiosos, sino que la construcción de conocimiento social y cultural se da en el intercambio de saberes entre el investigador y la comunidad; la comunidad es asumida como sujeto participante en el proceso investigativo, no como mero informante. La investigación busca la transformación social y política de la realidad de la comunidad. La pedagogía crítica de Paulo Freire está en total sintonía con esta apuesta.

El tercer movimiento fue una reafirmación de los principios de la ciencia moderna experimental pero con una peculiaridad: la reivindicación de su aplicación. Se trata del pragmatismo anglosajón que planteó que el conocimiento científico era aquel que generaba resultados eficaces y eficientes con base en unos objetivos preestablecidos. El pragmatismo permitió es el fundamento epistemológico del desarrollo vertiginoso de las ciencias aplicadas y la tecnología.

Esta tendencia también ha tenido efectos en la pedagogia: la tecnología educativa, que presupone que la enseñanza y el aprendizaje pueden ser eficaces y eficientes si se siguen unos pasos y procesos preestablecidos desde unos criterios de calidad y si se producen herramientas y mediaciones tecnológicas que mejorarán el proceso pedagógico. Esta es, desde mi punto de vista la perspectiva epistemológica hegemónica.

El cuarto y último momento es vigente y contemporáneo, desde fines del $\mathrm{s}$. XX: el cuestionamiento de la falta de interrelación entre las disciplinas, del aislamiento de cada experto en su campo de conocimiento; como alternativa aparecieron los conceptos interdisciplinariedad, multidisciplinariedad y transdsiciplinariedad, para justificar la necesidad de configurar redes comunicantes entre estas, que ha 
propiciado una cuarta circunstancia: que se eviten las categorías ciencia y disciplina y se prefiera una categoría parecida, aunque sin duda, radicalmente diferente: campos de estudio.

El campo de estudios es entendido como un ámbito de posibilidades para ejercer la investigación sobre un objeto de su preferencia; en este caso se pretende ir en contra de la categoría de ciencia como tal, se ignoran por completo las fronteras disciplinares y apelando al aporte o antecedente de cualquier tradición se plantea la posibilidad de que los presupuestos teóricos y metodológicos pueden configurarse en virtud de cruces entre campos diversos. En este caso, caso se busca fisurar la solidez que la categoria moderna de ciencia y asume que todo campo de estudios puede construir conocimiento riguroso en virtud de su desarrollo y en la apertura rigurosa a diversas tradiciones y saberes.

En este ámbito tiene cabida la investigación educativa, entendida no como ciencia, sino como un campo de estudios que puede beber de distintas disciplinas, sin temor a que esto la invalide.

\section{La pregunta por el conocimiento riguroso}

El debate sobre el término ciencia, permite intuir porqué al principio indiqué que la pregunta central de la epistemología es ¿Qué es el conocimiento, en el sentido más estricto y riguroso del término? ¿Cuáles son las condiciones de posibilidad de esa forma de conocimiento? En efecto, más que saber si algo es ciencia o no, de lo que se trata es que en medio de cualquier alternativa, nos encontramos, pese a las diferencias, con dos comunes denominadores: que sea lo que sea que se ocupe de conocer formalmente algo, ese algo debe ser configurado como objeto de estudio y que este ejercicio no puede hacerse de cualquier manera, sino que requiere de un talante conceptual y metódico denominado rigurosidad.

Así, el conocimiento riguroso es una construcción y construirlo demanda un esfuerzo; ese esfuerzo es la investigación. Desde los griegos hasta hoy se considera que no cualquier opinión o punto de vista es conocimiento, sino que requiere elaboración, decantación y fundamentación; esta fundamentación se ha hecho a partir de unas fuentes y de unos criterios de validez. Estos dos elementos son determinantes para cualquier investigación. Veamos: 


\subsection{Las fuentes del conocimiento riguroso}

En este aspecto ha habido dos fuentes principales para fundamentar el conocimiento riguroso: los sentidos o la experiencia y la racionalidad o los conceptos. Las fuentes derivadas de la experiencia pueden ser orales (sondeos de opinión orales, historias de vida), escritos (archivos históricos, sondeos de opinión escritos, pruebas y test estandarizados, encuestas estructuradas o semiestructuradas, diarios de campo), grabadas en recursos tecnológicos (videos, audios), objetos arqueológicos, (artefactos) o experimentales (experimentos de laboratorio).

Las fuentes derivadas de la racionalidad o los conceptos son las teorias existentes en un campo de estudios y que se encuentran en libros, artículos, ponencias, etc. y que van configurando conceptos a priori formales. Los conceptos a priori son categorías o conceptos previos que tenemos de las cosas; estos pueden ser informales (de la vida cotidiana) o formales (categorías de análisis de las disciplinas); son previos a la experiencia y tendemos a usarlos para caracterizar toda experiencia posible.

Ahora bien investigar puede hacerse confiando más en un tipo de fuente que en otro:

a. cuando la construcción de conocimiento se hace confiando más en las fuentes de la experiencia, los métodos preferidos son la experimentación y la inducción: es decir, se parte de experiencias particulares para a partir de ellas construir una teoria que generalice y explique todas las experiencias (síntesis, leyes). También métodos interpretativos en los que se pretende partir de los hechos sin conceptos a priori. No obstante, la crítica a esta tendencia es que cree que no parte de prejuicios ya que se funda en los hechos y presupone que los datos hablan por sí solos. Esto no es asi y un ejemplo de esto son los siguientes gráficos: 


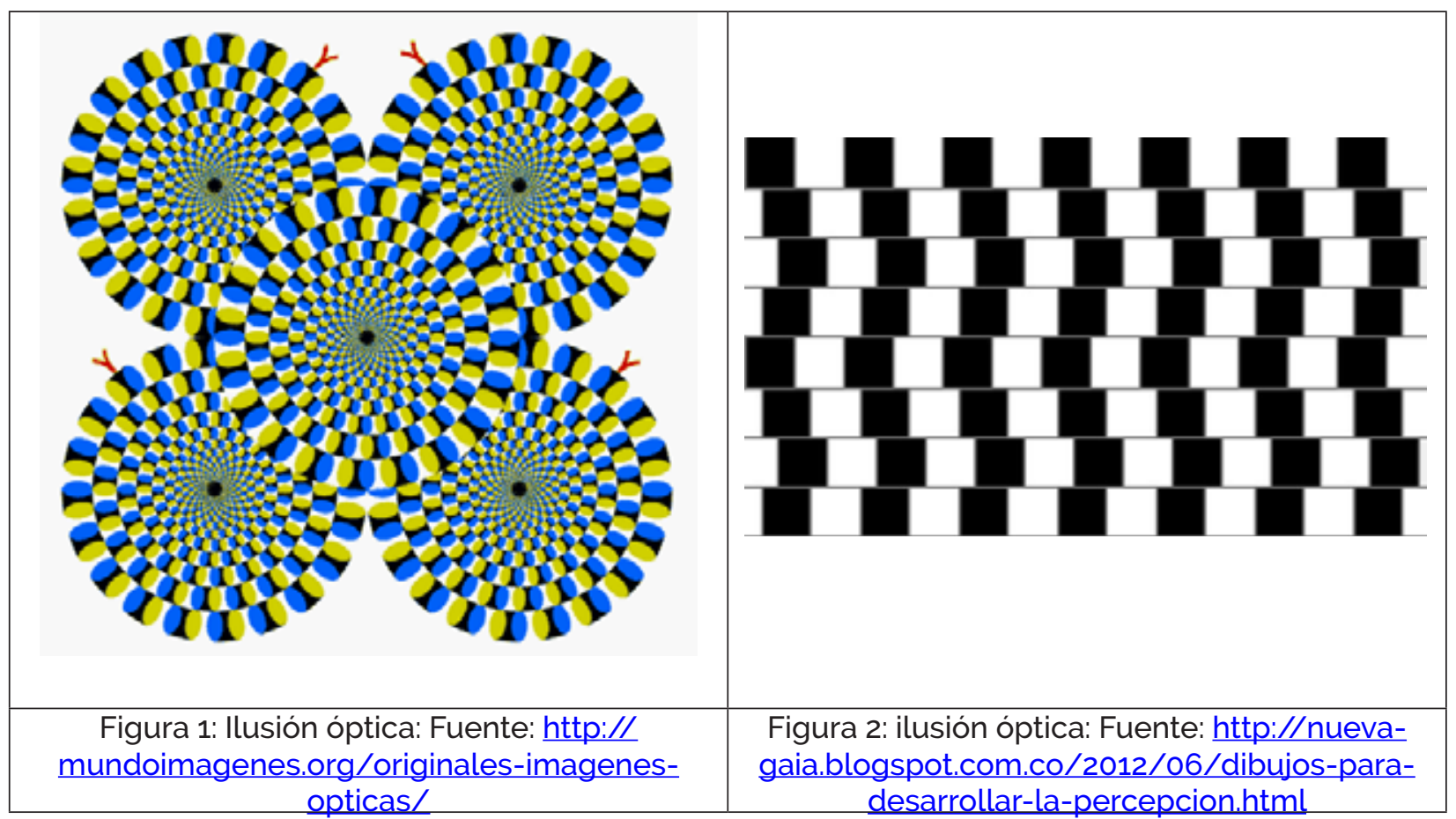

Si se observan detenidamente los gráficos, vemos que nuestra percepción no es tal cual vemos. En efecto, mientras en la figura 1 se tiene la sensación de movimiento lo cual no es real (se trata de una imagen estática), en la figura 2 parece que las líneas están torcidas cuando en realidad no lo están; esto sucede porque el entendimiento humano "hace hablar" a los datos por unas estructuras cognitivas. En la tercera imagen, el caricaturista Quino nos muestra cómo una misma imagen puede ser interpretada para seis propósitos distintos, todos ellos marcados por preferencias ideológicas diferentes.

De este modo, las investigaciones que pretenden confiar exclusivamente en los datos sin conceptos a priori corren el riesgo de dar por sentados los intereses que median sus análisis y los conceptos que subyacen a ello, asi como de estancar las maneras de problematizar los fenómenos.

\section{b. cuando la construcción de conocimiento se hace confiando más en las fuentes} de la razón y las categorias de análisis o conceptos a priori, los métodos preferidos son la deducción y los métodos interpretativos; en este caso, se da por sentado y se mira la realidad desde ese filtro; se presupone que la teoría es universal y válida per se, y desde ella se explican los fenómenos; en el fondo se asume la teoría como un dogma. 
Por ejemplo, cuando se lee el horóscopo y lo que se dice parece que se adecúa a lo que se está viviendo; en este caso, lo que está pasando es que el lector hace coincidir lo que vive con lo que lee, lo que da para que crea que el horóscopo sí dice algo cierto. Esto también pasa en investigación, cuando hacemos coincidir los datos con los prejuicios o conceptos a priori que tenemos: decir que los niños de 5 años están en retraso cognitivo si no se adecúan a lo que planteó Piaget en relación con esa edad.

Esto pone en evidencia que se hace necesaria una postura intermedia: una que asuma con igual importancia las dos fuentes de conocimiento: es decir que se reconozca que tenemos unos conceptos a priori que median el análisis de la experiencia, pero que esos conceptos tienen una historicidad; por ejemplo estudiar la infancia implica reconocer que el concepto infancia, no siempre ha sido pensado como lo pensamos hoy. Y también reconocer que de nada sirve quedarse en los conceptos si no se estudian los fenómenos.

Esto pone en evidencia que la construcción del conocimiento implica partir de unas teorias y conceptos que se someten a prueba con la experiencia (es decir, nos preguntamos: ¿la teorias que tenemos, los conceptos a priori formales, explican suficientemente los fenómenos?); pero al mismo tiempo la experiencia se somete a prueba con las teorías (o sea, nos preguntamos: ¿qué fenómenos pueden problematizarse a la luz de las teorias y conceptos a priori formales que tenemos?)

Esto nos ayuda a comprender dos cosas fundamentales de la construcción del conocimiento riguroso, es decir, de la investigación: La primera, que un problema de investigación no es una pregunta que se nos ocurre sin más ni más; la pregunta de investigación se construye en el seno de un campo de estudios específico que tiene sus conceptos a priori, sus categorias; el problema de investigación tiene en su interior varias categorias y subconceptos por caracterizar o relacionar.

Por eso un problema de investigación surge de la inmersión en el campo de estudios, es decir, de la revisión de unos antecedentes (de ahí la importancia del estado del arte) y de encontrar en ese campo un vacío, es decir, de caer en la cuenta de que hay una serie de experiencias o fenómenos que no han sido problematizados o no lo suficientemente en todas sus formas posibles. 
La segunda, que así como no cualquier pregunta es un problema de investigación, no cualquier respuesta es un conocimiento riguroso; no es un asunto de opinión o de punto de vista; llamamos investigación al esfuerzo de construcción de conocimiento mediado por unos referentes teóricos y metodológicos que demanda recursos espaciales, temporales, económicos y culturales. En otras palabras, investigar es asumir, mediante unos fundamentos epistemológicos (rigurosidad), un problema irresuelto - pese a unas experiencias de vida y unos conceptos o antecedentes-, configurándolo como objeto de estudio con el propósito de construir una teoria/práctica que solucione ese problema $\mathrm{o}$, al menos, lo esclarezca.

\subsection{Criterios de validez del conocimiento riguroso}

Ahora bien; la rigurosidad va siendo entendida de formas diferentes y hasta contrarias entre sí. Pudimos observar tres criterios de validez del conocimiento que perviven:

El criterio del enfoque empírico analítico, propio de la ciencia experimental moderna; la objetividad es el criterio, es decir, lo exacto y lo verificable;

El criterio del enfoque hermenéutico y del enfoque sociocrítico, propio de las ciencias interpretativas; en este caso el criterio no es la objetividad sino la validación verificable de los múltiples sujetos reconocidos como interlocutores válidos (ya vimos que no en todos los casos se reconoce a todos los sujetos) que intervienen en ese proceso, fruto de la interacción;

Y el criterio pragmatista, propio de las ciencias aplicadas; en este caso el criterio de validez es la eficacia o eficiencia, el alcance de unos objetivos preestablecidos, o buenos resultados;

Ahora bien; cada criterio de validez tiene unas implicaciones metodológicas ¿cuáles serian? 


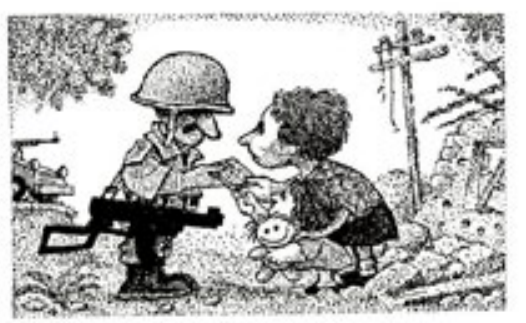

BOOROVOUN SOLDADO DCL EJTECITO QUE APOYA AL. DEMOCRATICO PRESIDENTE MAZZVICH REOALA GOCOLATE A LNA MADRE Y SU AIJITA ENTRE LAS

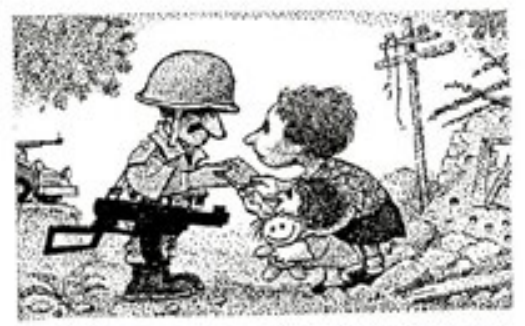

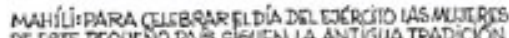

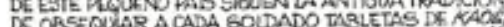

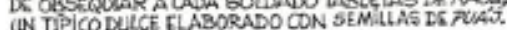

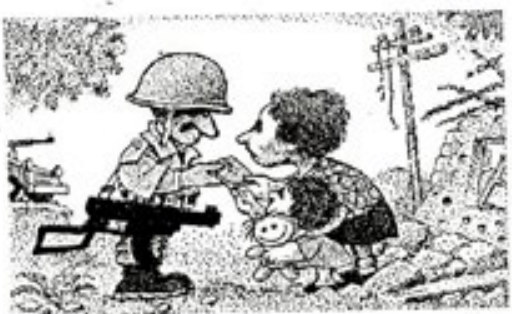

KÁFARA:UNA DEQUEÑA KAFREIZA DEIREGAALN INTEERANTEDELA MUCA VLNGADOKLSPORLAPAZ LN

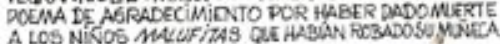

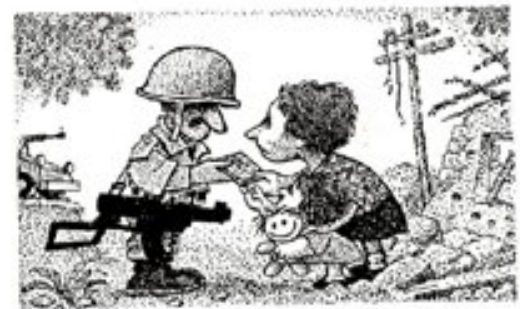

SAN OUANDE TAIPINGOLLIN AGENTE DEL CUERPO ANT NARCOTICOS CONTROLA LOS DOCUMENTOS DE UNA CAMPESINA. MUOASDE GLLAS UTILIZANA SUS Hi-

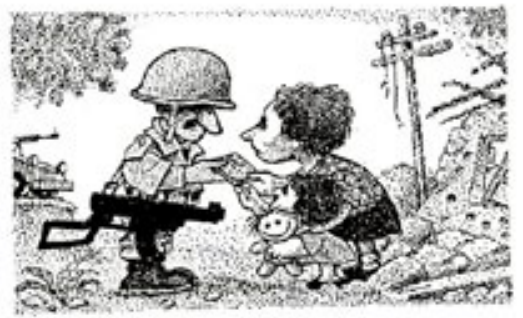

SINEBRA:UN WNFORNEDEL ENICET RIVLA QIE COSE EN WODOEL MUNDO EL NUMERO DE NINOS VITINASDC ABYSO

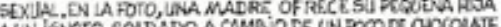
AUN IONOTO SOLDADO A CAMBIO DE UNFOCO DE CHOCOUTE.

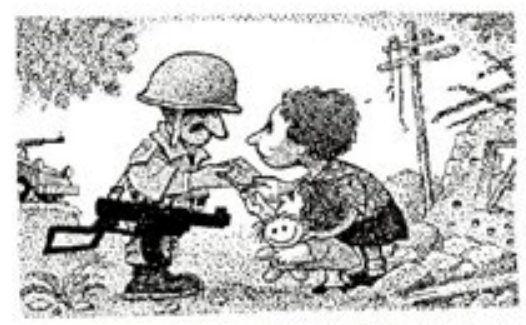

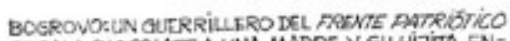
REGALA CHQCOLATE A UNA MADRE Y SU HIJTA EN RE LAS LUNAS DE SU CASA DESTRLDA TOR EL DARTIO QCE.AFONA AL SANGUINARO DI, MATEVICH.

Figura 3: Quino. Fuente: http://losparanoicos.blogspot.com.co/2011 0501 archive.html

\section{La pregunta por la construcción de conocimiento en el campo de la educación}

A lo largo de este texto se ha planteado que la epistemología es un campo de reflexión fundamental para la construcción del conocimiento. Después de haber analizado sus generalidades, nos compete analizar sus implicaciones en la construcción de conocimiento en el campo de la educación y la importancia de su determinación para su desarrollo.

Cuando la educación o los fenómenos educativos han sido asumidos como un 
objeto de investigación, la postura se ha bifurcado: por un lado, se ha asumido la educación como un objeto investigable por unas ciencias específicas (las ciencias de la educación, entre las cuales se encuentran la pedagogia, la psicopedagogía, la didáctica y las didácticas específicas); por el otro, se ha asumido como un objeto investigable por cualquier ciencia o campo de estudios interesado en él, como es el caso de la sociología, la filosofía, la historia, la antropología, de ahi que en el marco de estas ciencias sea posible adjuntarle la educación como un genitivo (sociología de la educación etc.).

Así, en libros (Abbagnano y Visalberghi, 1984; Restrepo, 1996; Martínez, 2000; Martínez-Boom, 2012; Noguera, 2012, Cristancho, 2013), y artículos de revistas (González, s.f.; Hostetler, 2007; Hernández, 2008; Rico, 2009; Galán y Zych, 2011; Pereira, 2011), se da por entendido educación como unos fenómenos relacionados con procesos de la enseñanza formal escolarizada de niveles diversos y que la investigación en educación se da en dicho ámbito.

No obstante, esta no es la única forma de asumir la cuestión. Como ya se mencionó en el primer ítem, se ha configurado un campo independiente pero transdisciplinar que es el de la investigación educativa; para entenderlo, es necesario tener en cuenta en qué ámbitos específicos de la educación se puede hablar de construcción de conocimiento.

Puede decirse que se construye conocimiento en la medida que los estudiantes y docentes van aprendiendo diversos conceptos, habilidades y formas de ser, que son objeto de la práctica pedagógica; por ejemplo, cuando un grupo de niños va aprendiendo las operaciones matemáticas como la suma y la resta, ellos van construyendo conocimiento matemático, al tiempo que el docente va construyendo conocimiento sobre cómo enseñarlo. Este primer ámbito es el pedagógico y didáctico y en este caso construir conocimiento es sinónimo de aprender y enseñar, aprender a enseñar y enseñar a aprender.

En segundo lugar, decimos que se construye conocimiento en la medida en que los directivos y la comunidad en general van asumiendo o construyendo directrices para orientar y dirigir los procesos administrativos y de convivencia de la comunidad 
educativa; por ejemplo, cuando los directivos y docentes van socializando y asimilando estándares del MEN, o van adaptando los PEl y manuales de convivencia a las necesidades que se van presentando; este segundo ámbito es organizativo y social y en este caso construir conocimiento es sinónimo de adaptarse a y adaptar el entorno social a unos fines específicos.

En tercer lugar, decimos que se construye conocimiento cuando el centro de enseñanza es, al mismo tiempo un centro de investigación, lo que implica que si cualquier miembro de la comunidad educativa desea saber algo, dispone de herramientas teóricas y metodológicas de campos de estudios. Por ejemplo, un docente y su grupo de estudiantes desean saber cuál es la historia de su localidad, región o municipio, o cuáles son los rasgos de la cultura política de esa región y emplean los conceptos y las técnicas de investigación propios de la historia o de la sociología para saberlo; o desean saber cuáles son los efectos en el medio ambiente y en las especies animales y vegetales nativas de las prácticas agrícolas de monocultivo que se han vuelto recurrentes en la región y emplean los conceptos y las técnicas de investigación propios de las ciencias naturales para saberlo; este tercer ámbito es el académico y en este caso construir conocimiento es sinónimo de investigar.

En sintesis, podemos afirmar que en el campo de la educación hay tres ámbitos 0 áreas de acción en los que se construye conocimiento, a saber, el pedagógico, el organizativo o social y el académico. Cada uno de ellos tiene su grado de complejidad; no obstante, cada uno de ellos puede relacionarse dialéctica y cíclicamente, lo cual implica las siguientes opciones:

a. Cuando el eje de referencia es el pedagógico:

a.b construir conocimiento es enseñar y aprender a investigar: cuando los estudiantes van aprendiendo los distintos conceptos de las disciplinas y las técnicas de investigación y los docentes van aprendiendo a enseñarla tenemos la pedagogía de la investigación; 
a.c construir conocimiento es enseñar y aprender a organizar y a dirigir: cuando estudiantes, docentes y directivos van aprendiendo reglas de juego de convivencia (a través de diversos mecanismos que incluyen distinciones, sanciones u otros) y van aprendiendo a configurarlas tenemos la pedagogía organizativa o social;

b. Cuando el eje de referencia es el organizativo tenemos las siguientes opciones:

b. a construir conocimiento es organizar los objetivos, pretensiones de las prácticas pedagógicas y los procesos de enseñanza y aprendizaje: en este caso tenemos la gestión u organización del currículo o gestión pedagógica;

b.c construir conocimiento es organizar la investigación, los procesos y procedimientos para asegurarla, administrarla y financiarla: en este caso tenemos la organización o gestión investigativa;

c. Cuando el eje de referencia es el académico tenemos las siguientes opciones:

c. a construir conocimiento es investigar las prácticas pedagógicas y los procesos de aprendizaje: en este caso tenemos la investigación pedagógica;

c.b construir conocimiento es investigar los procesos de organización social de las comunidades y la gestión académica y administrativa: en este caso tenemos la investigación de la organización y gestión educativa;

En términos conceptuales o abstractos, suponiendo que la construcción de conocimiento en estos tres campos se diera de manera fructífera, constante, y complementaria, cada una de ellas beneficiaria a la otra: la investigación pedagógica y la gestión pedagógica contribuirian a mejorar los procesos de enseñanza y aprendizaje; la pedagogía de la investigación y la gestión investigativa contribuirian a mejorar el diseño y la ejecución de proyectos de investigación; finalmente la investigación de la organización y gestión educativa y la pedagogía de lo social contribuirian a mejorar la organización, la convivencia y las relaciones sociales.

No obstante, existe siempre la posibilidad de que esto sea totalmente disperso e incompleto; por ejemplo, puede pasar que en una institución educativa la pedagogía 
se restrinja única y exclusivamente a la transmisión de las caracteristicas generales de los diversos campos del conocimiento; así, se puede enseñar y aprender historia, mas no necesariamente a ser historiador(a) (investigador(a) que reconstruye la historia), o enseñar y aprender ciencias naturales pero no necesariamente a ser investigador de la naturaleza; ejemplos parecidos pueden plantearse en los otros subcampos y en sus relaciones.

Lo anterior quiere decir que pese a que una institución educativa sea un espacio temporalidad para la construcción de conocimiento, de ahí no se deduce que toda institución educativa sea un centro de investigación pero si que podría serlo. Y esto, porque el eje que más fortalece a una institución educativa o a una comunidad es el de la investigación, porque esta permite problematizar lo ya sabido y profundizar en nuevas formas de comprender y de hacer las cosas.

Ahora bien, como puede verse, la investigación puede ejercerse en muchas disciplinas o campos de estudio: las ciencias formales (matemáticas), las ciencias naturales (biología, química, física, etc.), las ciencias aplicadas (ingenierias, tecnologías, etc.) y las ciencias sociales y humanas (lingüística, literatura, historia, filosofía, sociología, antropología, economía, etc.); en este último grupo ubicamos la investigación educativa, ya que es un campo de estudios que tiene que ver con el ser humano y lo social. No obstante, como lo indicamos arriba, este es un campo transdisciplinario.

Como se señaló arriba en la letra (c), en un centro educativo puede el área pedagógica y el área de la gestión y organización educativa; estas dos últimas, la investigación pedagógica y la investigación de la organización y gestión educativa conforman de manera específica el campo de estudios que nos interesa: el campo de investigación educativa.

Tomando en cuenta lo anterior, podriamos plantearnos las siguientes preguntas: ¿Qué tan fructíferas, constantes, y complementarias son las diversas formas de construcción de conocimiento en la institución educativa $X$ ? ¿Cuáles son las causas de que estas formas de construcción de conocimiento sean o no sean fructíferas, constantes, y complementarias en la institución educativa $X$ ? 
También podriamos hacer objeto de estudio de la investigación en educación una práctica pedagógica, una dificultad de aprendizaje, las maneras de situarse de los sujetos de una comunidad pedagógica (docentes, padres de familia, estudiantes, directivos), la convivencia de colegios públicos o privados, la situación financiera de las universidades públicas, o un fenómeno que aqueje una comunidad académica (el bullying, la violencia escolar, el bajo rendimiento académico, etc.).

Delmismo modo, tomando en cuenta otros campos de estudio, como por ejemplo, los estudios culturales, se puede ampliar el panorama de problemas de investigación: en primer lugar, la categoría educación entra en crisis porque para este campo, la educación ya no podrá sobreentenderse como el ámbito de los escenarios escolares formales, porque tendrá que ser asumida como una producción cultural.

De este modo, si bien los escenarios escolares podrán ser estudiados en clave cultural, no serán los únicos objetos posibles; de hecho, aparecerá la pregunta básica por cuáles son las prácticas y expresiones culturales que implícita e informalmente pueden ser pensadas como procesos educativos (ver anexo 1). Emergen por tanto, varios ejes de trabajo en los cuales pueden configurarse múltiples objetos de investigación (anexo 2).

Ya lo hemos indicado: frente a todos estos interrogantes podriamos arriesgar de manera desprevenida posibles respuestas; de hecho, es usual que lo hagamos, es decir que expresemos nuestra opinión o lo que nos dice el sentido común. Pero en este caso, estas preguntas tienen en su interior tantas categorias y subconceptos por caracterizar y relacionar, son lo suficientemente complejas que por eso merecen el nombre de problemas de investigación, pues para ser abordados no basta la espontaneidad de la opinión o del sentido común a la ligera; requieren el esfuerzo de construcción de conocimiento mediado por unos referentes teóricos y metodológicos y que demanda recursos espaciales, temporales, económicos y culturales; o sea, lo que llamamos investigación. 


\section{Bibliografía}

Abbagnano; Visalberghi. (1984). Historia de la pedagogía. Madrid: F.C.E.

Arendt, H. (1997). ¿Qué es la política? Barcelona: Paidós.

Arendt, H. (2008). La promesa de la política. Barcelona: Paidós.

Cristancho, J.G. (2013). Algunos diálogos sobre educación. Medellín: Universidad de Antioquia.

Foucault, M. (1998). Las palabras y las cosas. Una arqueología de las ciencias humanas. Madrid: Siglo XXI.

Galán, A; Zych, I. (2011). "Análisis de los criterios de la comisión nacional evaluadora de la actividad investigadora (CNEAl) para la concesión de los tramos de investigación en educación". En: Bordón 63 (2): 117-139.

González, J. (s.f.). El paradigma interpretativo en la investigación social y educativa. Nuevas respuestas para viejos interrogantes. Disponible en: http://institucional.us.es/ revistas/cuestiones/15/art_16.pdf

Hernández, F. (2008). "La investigación basada en las artes. Propuestas para repensar la investigación en educación". En: Educatio Siglo XXI, 26: 85-118.

Hostetler, K. (2007). Cómo debería ser una "buena" investigación en educación. Vestigium. Revista Académica Universitaria, 3 (1), 8-18.

Martínez-Boom, A. (2012). Verdades y mentiras sobre la escuela. Bogotá: IDEP.

Martínez, M. (2000). La investigación cualitativa etnográfica en educación. Manual teórico-práctico. México, D.F.

MEN (2015). Primer taller con universidades aliadas del programa de Becas para 
la excelencia docente: de la cobertura a la calidad. Bogotá, 27 y 28 de octubre. Documento de presentación y programación.

Noguera, C. (2012). El gobierno pedagógico. Del arte de educar a las tradiciones pedagógicas. Bogotá: Siglo Del Hombre Editores.

Pereira, Z. (2011). "Los diseños de método mixto en la investigación en educación: Una experiencia concreta". En: Revista Electrónica Educare. XV (1): 15-29. Disponible en http://www.revistas.una.ac.cr/index.php/EDUCARE/article/viewFile/867/793

Restrepo, B. (1996). Investigación en educación. Bogotá: ICFES.

Rico, L. (2009). "Sobre las nociones de representación y comprensión en la investigación en educación matemática". En: PNA, 4(1), 1-14. Disponible en http:// funes.uniandes.edu.co/662/1/Rico200gSobre.pdf 
Cirstancho Altuzarra J. (2019). La pregunta por los fundamentos epistemológicos de la investigación en la educación -aportes para una discusión epistemológica-.

Cuestiones De Filosofía, 5(24). 37-61

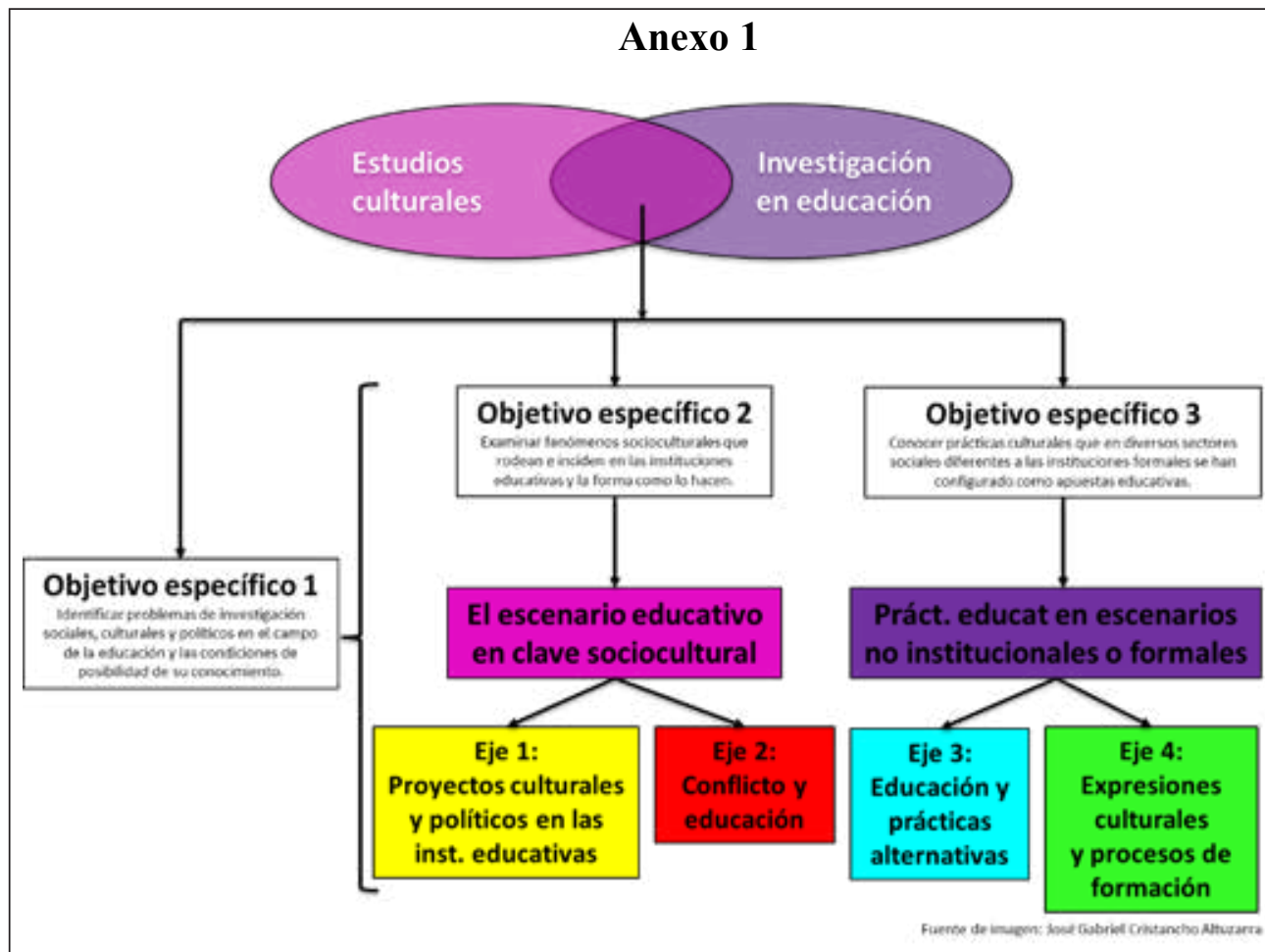

Anexo 2

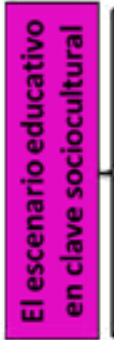

Eje 1: Proyectos

culturales y politicos

en las inst. educativas

¿cuáles proyectos culturales y politicos han sido fundamento de las apuestas educativas?

- ¿Qué tipo de sujetos pedagógicos se están configurando en virtud de las

politicas educativas orden nacional y global?

- ¿Qué rasgos culturales se han ido configurando en virtud de las prácticas

del sistema educativo formal?

- ¿Que proyectos culturales abanderan o lideran los maestros y maestras en sus instituciones?

Eje 2: Conflicto y educación $\left\{\begin{array}{l}\text { ¿Cómo inciden en las instituciones educativas fenómenos } \\ \text { socioculturales que afectan al país? } \\ \text { Desempleo, Desplazamiento forzado, Desmovilización, Violencla } \\ \text { social e intrafamiliar, Otros }\end{array}\right.$



\section{Eje 3: Educación y prácticas alternativas}

¿Cuáles son las caracteristicas de las prácticas culturales que en diversos sectores sociales se han configurado como apuestas educativas diferentes a las instituciones formales?

Sisternatizar la experiencia y las prácticas educativas de comunidades indigenas, afrodescendientes, campesinos, lideres sociales, etc.

Eje 4: Expresiones culturales ¿Qué procesos de formación se evidencian en diversas prácticas y y procesos de formación expresiones culturales contemporáneas como el cine, el teatro, los performances, los medios de comunicación social, entre otros? (Fenómenos estéticos y rituales, entre otros y su relación con la educación) 